\title{
Hugues Fontaine et Frédéric K. Panni (dir.), Des machines au service du peuple. Godin et la mécanique
}

Guise, Les éditions du Familistère, 2017, 160 p.

\section{François Jarrige}

\section{(2) OpenEdition}

Journals

Édition électronique

URL : http://journals.openedition.org/artefact/2524

DOI : $10.4000 /$ artefact.2524

ISSN : 2606-9245

Éditeur :

Association Artefact. Techniques histoire et sciences humaines, Presses universitaires du Midi

\section{Édition imprimée}

Date de publication : 6 décembre 2018

Pagination : 363-367

ISBN : 978-2-8107-0595-5

ISSN : 2273-0753

\section{Référence électronique}

François Jarrige, « Hugues Fontaine et Frédéric K. Panni (dir.), Des machines au service du peuple. Godin et la mécanique », Artefact [En ligne], 8 | 2018, mis en ligne le 21 juin 2019, consulté le 02 décembre 2020. URL : http://journals.openedition.org/artefact/2524 ; DOI : https://doi.org/10.4000/artefact. 2524

Ce document a été généré automatiquement le 2 décembre 2020.

Artefact, Techniques, histoire et sciences humaines est mise à disposition selon les termes de la Licence Creative Commons Attribution - Pas d'Utilisation Commerciale - Pas de Modification 4.0 International. 


\section{Hugues Fontaine et Frédéric K. Panni (dir.), Des machines au service du peuple. Godin et la mécanique}

Guise, Les éditions du Familistère, 2017, 160 p.

\section{François Jarrige}

1 Pour célébrer le bicentenaire de la naissance de Jean-Baptiste André Godin (1817-1888), le familistère de Guise a organisé dans ses locaux une belle exposition d'octobre 2017 à juin 2018. Sous le commissariat scientifique des conservateurs du patrimoine Claudine Cartier et Frédéric Panni, également directeur du Familistère, cent cinquante objets et pièces diverses ont été rassemblés. On y trouve des peintures, photographies ou gravures représentant des scènes du travail industriel, mais aussi des plans et maquettes de machines, ou des manuscrits inédits extraits des archives. L'ensemble dessine un parcours passionnant dans l'univers mécanique de Godin et la culture matérielle de son temps. Parallèlement, un ouvrage richement illustré, est publié qui reprend une sélection des œuvres exposées.

Cette réalisation s'inscrit dans le cadre du renouveau des activités du familistère depuis l'an 2000. Face à l'ampleur et à la richesse du patrimoine de ce lieu chargé d'histoire, et menacé d'une dégradation préoccupante depuis sa fermeture en 1968, le Conseil général de l'Aisne et la ville de Guise ont en effet créé en 2000 un syndicat mixte chargé de la valorisation du site ${ }^{1}$. Ce projet nommé «Utopia » devait à la fois restaurer et restructurer les bâtiments, créer un musée dédié au familistère et aux expérimentations sociales, et initier un lieu consacré aux utopies intellectuelles, culturelles ou artistiques. Le projet est ambitieux, il tente d'associer le passé et le présent, de dresser des ponts entre les aspirations réformatrices du XIX siècle, dont Godin est un symbole, et les enjeux contemporains d'un territoire en crise. Parallèlement ont été créées les éditions du Familistère qui ont notamment réédité des écrits de Godin, comme son livre Solutions sociales paru en 1871. Dans ce long texte rédigé avant la Commune mais publié juste après, Godin proposait une synthèse de ses projets et réalisations, il y exposait sa vision du monde, ses aspirations politiques et 
sociales. Dans la foulée du fouriérisme, les réformes sociales étaient pensées comme des instruments de pacification chargés de conjurer « la guerre civile».

Godin demeure une figure fascinante, à de nombreux égards ambivalente, qui incarne les doutes et les tensions qui traversent les sociétés du XIX siècle à propos du phénomène de la grande industrie et du machinisme conquérant. À la différence du maitre Charles Fourier, mort en 1837, et contemporain d'un monde essentiellement rural et artisanal, Godin incarne la génération suivante des réformateurs sociaux, totalement plongée dans le bain de l'industrialisme et la fascination pour le progrès technique. Il serait simpliste de ramener Godin à l'utopie et au "socialisme", sa trajectoire est celle d'un petit industriel contemporain des rapides mutations intellectuelles, politiques et économiques du milieu du xix ${ }^{e}$ siècle. Il appartient à cette génération de militants fouriéristes déçus par la Révolution de 1848 et ses échecs, frustrés par les expériences ratées de réalisation du Phalanstère, contraints à la modération politique par l'Empire autoritaire.

Fils d'un simple artisan serrurier, contraint d'arrêter très jeune l'école, Godin apprend le travail des métaux et réalise un tour de France qui le met en contact avec la diversité des mondes du travail. En 1840, il monte un atelier de construction d'appareils de chauffage et obtient le 15 juillet 1840 le brevet de fabrication d'un poêle en fonte qui fera son succès et sa richesse. Godin est donc un artisan habitué à manier la matière, à utiliser des outils pour fabriquer des équipements complexes au moment où la construction mécanique se développe et se spécialise. C'est également à cette époque qu'il découvre le fouriérisme à travers un article publié dans la presse locale. Les disciples de Fourier menés par Victor Considerant cherchent alors à élargir leur audience en publiant de nombreux journaux et brochures. Comme beaucoup dans cette génération qui s'est politisée dans la fièvre intellectuelle des années 1840, Godin se tourne alors vers la question sociale, lit les utopistes, et communie dans le culte de la fraternité et de l'association ouvrière, censée réconcilier les progrès matériels et sociaux. Comme les autres fouriéristes, souvent des notables ou de petits industriels, il se méfie en revanche des actions révolutionnaires et répugne aux doctrines communistes et aux théories radicales qui remettent en cause la propriété privée.

Godin entend d'abord réconcilier le travail et le capital, instaurer une troisième voie pacifique entre l'exploitation libérale et le collectivisme. Comme beaucoup de ses contemporains, son projet vise aussi à sauver le progrès technique de sa captation par le libéralisme égoïste, en imaginant des usages sociaux émancipateurs des développements de l'industrie et de la mécanique, perçus de plus en plus comme des trajectoires inéluctables et bénéfiques ${ }^{2}$. En 1846, Godin transfère sa fonderie à Guise, au bord de l'Oise, la petite manufacture de poêles ne compte encore qu'une trentaine d'ouvriers. Sa réussite économique démarre surtout sous le second Empire dans une période de forte croissance et d'industrialisation du pays, lorsque l'essor des niveaux de vie favorise l'achat d'équipements de confort moderne comme ses poêles. Après l'expérience malheureuse du phalanstère de La Réunion, au Texas, en 1855 où il perd une partie de sa fortune, Godin abandonne les expériences collectives et tente de réaliser ses projets de réformes sociales à partir du site de Guise. Sensible à l'idée de redistribution des richesses aux ouvriers, il souhaite créer une alternative à la société industrielle libérale et offrir aux ouvriers le confort dont seuls les bourgeois pouvaient alors bénéficier. La construction du Familistère de Guise, qui s'étend de 1859 à 1884, répond à ce projet. L'activité de l'usine se développe et le nombre d'ouvriers explose. 
Godin met sur pied un système d'assistance sociale autour de son usine, largement inspiré par les préceptes fouriéristes expurgés de ce qu'ils avaient de plus subversifs. Il construit ainsi le Palais social comprenant des logements modernes pour l'époque; il multiplie les services pour ses ouvriers (économat, crèche, lavoir, piscine...), développe des activités culturelles (écoles, cours du soir pour les adultes, théâtre...) ainsi que divers avantages sociaux pour la main-d'œuvre (assurance maladie, retraite, etc.). Son projet est contemporain de l'essor des idées et pratiques paternalistes qui cherchent à concilier la grande industrie et la pacification des rapports sociaux.

Godin se moquait souvent des "philanthropes », pour lui le Familistère ne devait pas seulement être un milieu confortable, il devait aussi préparer les esprits à l'association en forgeant une émancipation collective, fondée sur l'unité sociale, voire spirituelle. En 1880 il crée l'association coopérative du capital et du travail, grâce à laquelle les travailleurs deviennent les capitalistes du Familistère et de ses usines. Mais ces réformes et ce nouveau milieu de vie émancipé ne pouvaient réellement fonctionner que sur la base de la réussite économique et des progrès de l'industrie et des machines. Comme Godin l'écrit en 1871 dans ses Solutions sociales, ce sont « les moyens nouveaux de production (qui) créent le développement de la richesse indispensable à la réalisation du bien-être de l'humanité et rendent déjà accessibles aux masses, des étoffes, des denrées, des objets de consommation de toute espèce ». Pour Godin, la modernité industrielle et technique, devenue le moteur de l'histoire, peut et doit être mise au service du peuple. Comme il l'écrit encore en 1886 dans une lettre: "La puissance des machines mise au service des associations productives créera le bien-être et l'aisance au profit des masses laborieuses». Cette ambition est au cœur de l'exposition organisée au Familistère, comme du livre qui l'accompagne. En quatre chapitres très intéressants, l'ouvrage restitue ce projet de Godin en explorant d'abord le nouveau milieu technique qui surgit au milieu du XIXe siècle avec le triomphe de la vapeur, l'accroissement des mécaniques pour réaliser toute une série de tâches industrielles ou domestiques. Le second chapitre est consacré au "Chemin de fer, figure de la transformation sociale ». Godin est en effet contemporain de l'essor ferroviaire. Après les débats qui ont lieu autour de 1840, la vision saint-simonienne s'impose rapidement et le chemin de fer devient l'objet d'une vive ferveur au fur et à mesure de sa démocratisation, il symbolise l'égalité en marche, l'essor des communications censé favoriser la paix. Grâce à de belles illustrations l'ouvrage montre bien comment le train envahit peu à peu les imaginaires. Les chapitres 3 et 4 se tournent plus nettement vers Godin à proprement parler en examinant quelques " machines admirées par Godin », ou du moins dont on peut supposer que Godin les admirait, celles qu'il a par exemple pu observer lors des expositions universelles comme les nouveaux appareils de communication à distance et d'imprimerie, ou les premiers moteurs électriques. Enfin, le dernier chapitre, plus court, présente les «machines imaginées par Godin ». À la différence des penseurs phalanstériens de la monarchie de Juillet qui se contentèrent d'imaginer des palais sociaux intégralement mécanisés et automatisés, Godin tenta de réaliser ce projet à la fois social et technique en équipant le Familistère des dernières innovations, comme des berceaux pour les crèches, des systèmes de ventilation, mais aussi de nombreux procédés censés accroitre la productivité des ateliers. Godin lui-même déposa des dizaines de brevet et, comme entrepreneur, il fut particulièrement soucieux de mécaniser les diverses opérations de son usine. Il se moula sans difficulté dans le nouvel imaginaire hérö̈que des techniques qui s'impose à son époque. 
$7 \mathrm{Au}$ fond, ce petit livre magnifiquement illustré nous plonge au cœur des représentations ambivalentes $d u$ progrès et de ses réalisations mécaniques au $\mathrm{XIX}^{\mathrm{e}}$ siècle. À travers Godin, on suit comment les aspirations radicales de transformation sociales, si vives avant 1848 , se sont mues après 1850 en accommodements et en expérimentations plus réduites. On suit comment la fascination pour la technique a peu à peu pris le pas. Mais l'aspiration à réconcilier progrès technique et progrès social et le désir de mettre les machines au service du peuple se heurte évidemment à des impasses et de nombreuses difficultés. Au xix ${ }^{e}$ siècle, Guise apparaît ainsi comme un îlot dans un univers d'exploitation. Par ailleurs au sein même des ateliers de Guise, les frictions et tensions pouvaient exister. Ainsi lorsque Godin introduit la mécanisation du travail du moulage dans ses ateliers, il espère une production de 300 châssis par jour. Mais seuls 80 sortent des ateliers, et les ouvriers s'étonnent des cadences exigées par le patron ${ }^{3}$. Car au fond qu'est-ce qu'une machine mise au service du peuple ? Comment faire la part entre les technologies pensées pour accroitre les profits et le contrôle en accentuant la dépossession et celles qui permettent de renforcer l'autonomie et la liberté des producteurs? Qu'est ce qui différencie des machines pour le peuple et celles qui vont contre ses intérêts? Ces questions furent vives au début de l'ère industrielle, elles modelèrent les imaginaires réformateurs et socialistes du temps. Elles restent évidemment plus que jamais d'actualité.

\section{NOTES}

1. Sur l'histoire du Familistère de Guise après la mort de son fondateur en 1888, et jusqu'à sa fermeture en 1968, voir Jessica Dos SANTOS, L'utopie en héritage. Le Familistère de Guise (1888-1968), Tours, Presses universitaires Francois-Rabelais, 2016.

2. François JARRIGE (dir.), Dompter Prométhée. Technologies et socialismes à l'âge romantique (1820-1870), Besançon, Presses universitaires de Franche Comté, 2016.

3. Michel lallement, Le travail de l'utopie. Godin et le familistère de Guise, Paris, Les Belles lettres, 2009, p. 336-337.

\section{AUTEUR}

FRANÇOIS JARRIGE

Université de Bourgogne, Centre Georges-Chevrier (UMR 7366) 